\title{
Caracterização e estimativa da variabilidade genética de genótipos de cebola
}

\author{
Gerson Henrique Wamser ${ }^{1}$; Bruna Arruda ${ }^{1}$; Jussara Cristina Stinghen ${ }^{1}$; Diane S Rozzetto ${ }^{1}$; Juliano G \\ Bertoldo $^{2}$; Sergio D Lannes ${ }^{3}$; Altamir Frederico Guidolin'; Jefferson Luís M Coimbra ${ }^{1}$ \\ 'UDESC-Depto. Agronomia, Av. Luiz de Camões 2090, Conta Dinheiro, 88520-000 Lages-SC; gwamser@epagri.sc.gov.br; bru_agro@, \\ hotmail.com; jujustinghen@gmail.com; dsrozzetto@gmail.com; altamirguidolin@gmail.com; coimbrajefferson@cav.udesc.br; ${ }^{2}$ UFSC, \\ Rod. Admar Gonzaga 1346, 88034-001 Florianópolis-SC; jgbertoldo@gmail.com; ${ }^{3}$ Epagri, R. Carlos Thiesen 75, Gabiroba, 88400-000 \\ Ituporanga-SC; sergiolannes@epagri.sc.gov.br
}

\begin{abstract}
RESUMO
Este trabalho teve como objetivo caracterizar genótipos de cebola cultivados em Santa Catarina e estimar a variabilidade genética existente entre os mesmos. Para isto foram avaliados quinze genótipos de cebola em dois ambientes, Ituporanga e Lages. O delineamento utilizado foi de blocos casualizados, com três repetições em cada ambiente. Foram avaliados o comprimento do pseudocaule; número de folhas por pseudocaule; diâmetro do pseudocaule; diâmetro do bulbo; altura do bulbo; peso do bulbo; relação altura:diâmetro do bulbo; produção total de bulbos; formato do bulbo; porcentagem de florescimento e porcentagem de bulbos podres. Os dados foram submetidos à análise de variância multivariada. Houve efeito significativo para a interação genótipos $\mathrm{x}$ ambientes, fato que causou diferenças nos valores de dissimilaridade em cada local. Foi elaborada uma matriz de dissimilaridade utilizando a distância de Mahalanobis. Os caracteres morfológicos e agronômicos utilizados foram suficientes para caracterizar os genótipos, indicando que os programas de melhoramento dispõem de uma ampla base genética para o desenvolvimento de novas cultivares.
\end{abstract}

Palavras-chave: Allium cepa, análise de variância multivariada, dissimilaridade, distância de Mahalanobis.

\begin{abstract}
Characterization and estimation of genetic variability of onion genotypes

This study aimed to characterize onion genotypes grown in Santa Catarina state, Brazil and to estimate their genetic variability. Fifteen onion genotypes were evaluated in two locations, Ituporanga and Lages. The experimental design was of randomized blocks with three replications in each environment. We evaluated the length of the pseudostem, number of leaves per pseudostem, stem diameter, bulb diameter, height of the bulb, bulb weight, height:diameter ratio; total production of bulbs, bulb shape, flowering percentage and percentage of rotten bulbs. The data were subjected to multivariate analysis of variance. The results showed significant effects for genotype-environment interaction, fact that was reflected in the values of dissimilarity in each location. A matrix of dissimilarity was prepared using Mahalanobis distance method. The morphological and agronomic traits used were sufficient to characterize the genotypes, indicating that breeding programs have a wide genetic base for the development of new cultivars.
\end{abstract}

Keywords: Allium cepa, multivariate analysis of variance, dissimilarity, Mahalanobis distance.

(Recebido para publicação em 29 de julho de 2011; aceito em 26 de abril de 2012) (Received on July 29, 2011; accepted on April 26, 2012)

\begin{abstract}
A cebola é uma das plantas cultivadas de mais ampla difusão no mundo, sendo consumida por quase todos os povos do planeta, independente da origem étnica e cultural, constituindo-se em importante elemento de ocupação da mão de obra familiar (Boiteux \& Melo, 2004). O Estado de Santa Catarina destaca-se como maior produtor nacional, com uma área plantada na safra 2009/2010 de 21.271 ha e uma produção de 454 mil toneladas, seguido pelos estados da Bahia e de São Paulo (Schmitt, 2010).

A maior parte da cebola cultivada hoje no estado é oriunda de cultivares de polinização aberta de ciclo precoce
\end{abstract}

a médio, lançadas há mais de 10 anos pela Empresa de Pesquisa Agropecuária e Extensão Rural de Santa Catarina (Epagri), como por exemplo Empasc 352 Bola Precoce, Empasc 355 Juporanga, Epagri 362 Crioula Alto Vale e Epagri 363 Super Precoce. A utilização da variabilidade genética é fundamental para os programas de melhoramento (Borém \& Miranda, 2009; Heidemann et al., 2010), pois tende a reduzir a vulnerabilidade das culturas a estresses bióticos e abióticos e, ao mesmo tempo, pode acelerar o progresso genético para determinados caracteres (Cui et al., 2001). A caracterização fenotípica dos genótipos constitui um importante subsídio para a escolha de genitores divergentes e complementares para o desenvolvimento de populações segregantes em programas de melhoramento genético de cebola, visando à diversificação do mercado de cultivares (Buzar et al., 2007). Quanto mais contrastantes forem os genitores dentro de um mesmo pool gênico, maior a variabilidade resultante na população segregante (Cruz \& Regazzi, 2001; Moura et al., 1999).

Vários autores têm utilizado descritores morfológicos de caráter métrico na caracterização da variabilidade de diferentes germoplasmas, (Buzar et al. (2007) em cebola; Cui et al. (2001) em soja; Mariot et al. (2008) em 
espinheira-santa). Barbieri et al. (2005) observaram que os caracteres que mais contribuíram para a divergência entre acessos de cebola são a cor da casca, o peso e a conservação pós-colheita dos bulbos e que o uso de caracteres morfológicos são suficientes para diferenciar as populações de cebola.

Para analisar a contribuição dos diversos descritores agronômicos na determinação da divergência genética pode ser adequado utilizar técnicas de análise multivariada. Este tipo de análise consiste na avaliação simultânea de vários caracteres e permite diferentes abordagens sobre os dados analisados, possibilitando esclarecer tanto a relação quanto o efeito de cada variável separadamente (Ferreira, 1996; Coimbra et al., 2007). As técnicas multivariadas mais utilizadas são as de variáveis canônicas, dos componentes principais e das distâncias euclidiana e de Mahalanobis (Cruz \& Carneiro, 2003).

O objetivo deste trabalho foi caracterizar genótipos de cebola cultivados em Santa Catarina e estimar a variabilidade genética existente entre os mesmos.

\section{MATERIAL E MÉTODOS}

Foram conduzidos dois experimentos na safra 2009/2010 em dois ambientes, sendo estes: estação experimental da Epagri de Ituporanga $\left[27^{\circ} 25^{\prime} \mathrm{S}\right.$, 49 $38^{\prime} \mathrm{W}$, altitude de $475 \mathrm{~m}$, clima subtropical úmido (Cfa) (classificação de Köppen)] e estação experimental da Epagri de Lages [27\%49'S e 50¹9'W, altitude de $913 \mathrm{~m}$, clima temperado úmido $(\mathrm{Cfb})]$. Foram avaliados quinze genótipos de cebola cultivados no estado de Santa Catarina, sendo assim categorizados: SuperSuper Precoce, Bela Vista, Baia Indaial, Crioula Roxa e Crioula Branca (populações mantidas pela Epagri); Empasc 352 Bola Precoce, Empasc 355 Juporanga, Epagri 362 Crioula Alto Vale e Epagri 363 Super Precoce (populações comerciais da Epagri); Bella Catarina, Bella Vista e Bella Dura (híbridos comerciais da Sakata); Boreal e Gauchinha (populações comerciais da Hortec); Catarina (população comercial da Agritu).

A condução dos experimentos seguiu as recomendações técnicas para a cultura (Epagri, 2000). O delineamento utilizado para ambos os experimentos foi de blocos casualizados com três repetições. As parcelas foram formadas por cinco linhas com três metros de comprimento, utilizando o espaçamento de 0,40 m entre linhas, com dez plantas por metro linear, totalizando 150 plantas por parcela. A área útil consistiu das três linhas centrais da parcela, descartando-se duas plantas de cada extremidade. A colheita foi realizada quando $60 \%$ das plantas haviam tombado, sendo que os bulbos foram deixados por aproximadamente quinze dias a campo para a secagem das folhas (cura) e a seguir depositados em armazém convencional, na estação experimental da Epagri de Ituporanga.

Foram avaliados: $i$ ) comprimento do pseudocaule (medida em centímetros da parte superior do bulbo até o final da última bainha foliar); ii) número de folhas por pseudocaule (contagem do número de folhas existentes); iii) diâmetro do pseudocaule (medida em milímetros da secção transversal do pseudocaule na sua porção central); iv) diâmetro do bulbo (medida em centímetros da maior secção transversal do bulbo); v) altura do bulbo (medida em centímetros da maior secção longitudinal do bulbo); vi) peso do bulbo (gramas da massa fresca total do bulbo); vii) relação altura:diâmetro do bulbo (razão entre a altura do bulbo e o diâmetro); viii) produção total de bulbos (produto entre o peso médio dos bulbos e o número de bulbos por parcela); $i x$ ) forma do bulbo (avaliação visual de acordo com formatos propostos nos descritores); $x$ ) porcentagem de florescimento (relação entre o número de plantas florescidas e o número total de plantas por parcela) e $x i$ ) porcentagem de bulbos podres (relação entre o número de bulbos podres e o número total de plantas por parcela). Estas características foram avaliadas de acordo com os descritores propostos pelo International Plant Genetic Resources Institute (IPGRI, 2001).

Como regra geral para a análise multivariada, a equação do modelo estatístico tem a mesma expressão da equação para a situação univariada, com os termos escalares substituídos por vetores, onde cada um dos termos é um vetor de dimensão "v" cujos elementos são identificados pelo mesmo símbolo do fator.

Tabela 1. Contrastes multivariados para 15 genótipos de cebola quando comparados em dois ambientes (Ituporanga e Lages); valor obtido pela estatística de Wilks, denominada de U e probabilidade de erro a 5\% de significância pelo teste $\mathrm{F}$ (multivariate contrasts for 15 onion genotypes when compared in two environments (Ituporanga and Lages), obtained by the Wilks statistic, named U and error probability by F test at 5\%). Lages, UDESC/Epagri, 2011.

\begin{tabular}{llll}
\hline Contrastes $^{*}$ & \multicolumn{1}{c}{ Genótipos } & Valor & Pr $>\mathbf{F}$ \\
\hline $\mathrm{C}_{1}:$ & SuperSuper Precoce & 0,5198 & 0,0006 \\
$\mathrm{C}_{2}:$ & Bela Vista & 0,3184 & 0,0001 \\
$\mathrm{C}_{3}:$ & Superprecoce & 0,3129 & 0,0001 \\
$\mathrm{C}_{4}:$ & 0,3511 & 0,0001 \\
$\mathrm{C}_{5}:$ & Baia Indaial & 0,4748 & 0,0001 \\
$\mathrm{C}_{6}:$ & Bola Precoce & 0,3374 & 0,0001 \\
$\mathrm{C}_{7}:$ & Juporanga & 0,3095 & 0,0001 \\
$\mathrm{C}_{8}:$ & Crioula & 0,3469 & 0,0001 \\
$\mathrm{C}_{9}:$ & Bella Catarina & 0,3211 & 0,0001 \\
$\mathrm{C}_{10}:$ & Bella Vista & 0,2426 & 0,0001 \\
$\mathrm{C}_{11}:$ & Bella Dura & 0,4773 & 0,0001 \\
$\mathrm{C}_{12}:$ & Boreal & 0,3873 & 0,0001 \\
$\mathrm{C}_{13}:$ & Catarina & 0,6456 & 0,0245 \\
$\mathrm{C}_{14}:$ & Crioula Roxa & 0,6974 & 0,0790 \\
$\mathrm{C}_{15}:$ & Crioula Branca & 0,7466 & 0,1971 \\
\hline
\end{tabular}

${ }^{*}$ Cada genótipo foi testado em cada ambiente (each genotype was tested in every environment), Ituporanga e Lages. 
$Y_{i j k}=\mu+a_{i}+b_{j}+a b_{i j}+\beta_{k}+e_{i j k}$

Onde: $\mu$ é a média geral esperada, $a_{i}$ é o efeito diferencial do nível $i$ do fator $\mathrm{A}, b_{j}$ é o efeito diferencial do nível $j$ do fator $\mathrm{B}, a b_{i j}$ é o efeito diferencial da interação do i-ésimo nível do fator A com o j-ésimo nível do fator $\mathrm{B}, \beta_{k}$ é o efeito de blocos e $e_{i j k}$ é o erro experimental. Os dados foram submetidos à análise de variância multivariada (MANOVA) com o auxílio do pacote estatístico SAS ${ }^{\circledR}$ 9.1.3 (2007).

\section{RESULTADOS E DISCUSSÃO}

O resultado da verificação de homogeneidade da matriz de covariância por meio da estatística de verossimilhança generalizada justifica o procedimento dos testes para situação da matriz de covariância heterogênea. A partir da análise da variância multivariada (dados não apresentados), pode ser observado que o efeito da interação genótipo x local foi significativo pelo teste de $\mathrm{F}$ a $5 \%$ de probabilidade de erro, evidenciando um comportamento diferenciado dos genótipos frente aos ambientes avaliados. Este efeito da interação pode inclusive levar diferentes genótipos avaliados a uma enorme diversidade de padrões e resultados (Coimbra et al., 2009). Portanto, é importante que se conheça o quanto o ambiente influencia na expressão das características sob avaliação. De fato é observado, na cultura da cebola, que o seu desenvolvimento é altamente dependente do clima, principalmente de fatores como o fotoperíodo e a temperatura. O produto final da cultura é o bulbo, sendo que o início da bulbificação ocorre quando as condições de luminosidade e temperatura ambiente são adequadas, ou seja, fotoperíodo entre 12 e 14 horas e temperatura entre 15 e $25^{\circ} \mathrm{C}$ para as condições do Brasil (Oliveira et al., 2004). Em situações em que estes fatores não atendam as necessidades da cultura, pode não ocorrer a formação do bulbo e consequentemente inviabilizar o cultivo. Portanto, é fundamental que os experimentos de seleção e avaliação de genitores elite sejam feitos na região onde a nova variedade será cultivada (Leite, 2007).

Para verificar o comportamento dos genótipos frente aos locais avaliados, foram realizados contrastes multivariados. Os resultados evidenciaram que todos os contrastes foram significativos, com exceção do $\mathrm{C}_{14}$ (contraste do genótipo Crioula Branca nos dois ambientes) e

Tabela 2. Coeficientes canônicos padronizados $(\mathrm{CCP})$ e a estatística da razão de verossimilhança $(\Lambda)$ das onze variáveis respostas avaliadas nos genótipos de cebola estudados, separadamente para cada um dos quinze contrastes testados $\left(\mathrm{C}_{1} \text { a } \mathrm{C}_{15}\right)^{*}$ (standardized canonical coefficients $(\mathrm{CCP})$ and the statistical likelihood ratio $(\Lambda)$ of the eleven variables measured in studied onion genotypes, separately for each of the fifteen tested contrasts). Lages, UDESC/Epagri, 2011.

\begin{tabular}{|c|c|c|c|c|c|c|c|c|c|c|c|c|}
\hline \multirow{2}{*}{ Contrastes $^{2}$} & \multicolumn{11}{|c|}{ Variáveis $^{1}$} & \multirow{2}{*}{$\Lambda$} \\
\hline & $\mathbf{a}$ & b & c & d & e & f & g & $\mathbf{h}$ & i & $\mathbf{j}$ & 1 & \\
\hline $\mathrm{C}_{1}$ & $-0,57$ & 2,40 & $-0,30$ & $-1,40$ & 2,66 & $-2,38$ & $-2,16$ & 0,91 & 0,20 & 1,23 & $-0,08$ & $0,51^{*}$ \\
\hline $\mathrm{C}_{2}$ & 0,32 & 1,92 & $-0,35$ & $-0,59$ & 0,16 & $-0,01$ & $-0,52$ & 0,53 & 0,32 & 1,09 & 0,26 & $0,31^{*}$ \\
\hline $\mathrm{C}_{3}$ & $-1,08$ & 3,42 & $-0,14$ & $-4,89$ & 4,06 & 0,61 & $-3,16$ & $-0,29$ & 0,24 & 0,39 & 0,77 & $0,31^{*}$ \\
\hline $\mathrm{C}_{4}$ & $-0,98$ & 3,59 & $-0,45$ & $-4,10$ & 3,43 & 0,66 & $-2,50$ & $-0,19$ & 0,26 & 0,17 & 0,84 & $0,35^{*}$ \\
\hline $\mathrm{C}_{5}$ & $-0,27$ & 2,83 & $-0,42$ & $-3,34$ & 2,41 & 1,36 & $-2,25$ & $-0,63$ & 0,26 & 0,72 & 0,38 & $0,48^{*}$ \\
\hline $\mathrm{C}_{6}$ & $-0,73$ & 3,26 & $-0,26$ & $-4,28$ & 1,53 & 4,44 & $-1,25$ & $-1,15$ & 0,17 & $-1,01$ & 0,48 & $0,33^{*}$ \\
\hline $\mathrm{C}_{7}$ & $-0,79$ & 3,73 & $-0,46$ & $-5,39$ & 2,90 & 3,28 & $-2,38$ & $-0,83$ & 0,08 & $-1,09$ & 0,59 & $0,30^{*}$ \\
\hline $\mathrm{C}_{8}$ & 0,32 & 3,59 & $-0,04$ & $-2,31$ & 1,31 & 0,75 & $-1,04$ & $-1,08$ & $-0,00$ & 0,01 & 0,33 & $0,34^{*}$ \\
\hline $\mathrm{C}_{9}$ & 0,29 & 3,05 & 0,45 & $-1,22$ & 0,18 & 1,25 & $-0,48$ & $-0,72$ & 0,41 & $-0,32$ & 0,17 & $0,32^{*}$ \\
\hline $\mathrm{C}_{10}$ & $-0,57$ & 2,83 & $-0,55$ & $-5,26$ & 3,10 & 3,59 & $-2,39$ & $-1,22$ & 0,27 & $-0,03$ & 1,06 & $0,24^{*}$ \\
\hline $\mathrm{C}_{11}$ & 0,65 & 3,17 & $-0,89$ & 3,36 & $-2,24$ & $-0,26$ & 1,68 & 0,52 & $-0,11$ & $-0,47$ & 0,35 & $0,47^{*}$ \\
\hline $\mathrm{C}_{12}$ & 0,20 & 3,16 & $-0,66$ & $-1,72$ & 0,18 & 3,17 & $-0,44$ & $-0,79$ & $-0,08$ & $-0,44$ & 0,41 & $0,38^{*}$ \\
\hline $\mathrm{C}_{13}$ & 0,16 & $-2,63$ & 0,42 & 1,43 & $-0,86$ & $-0,34$ & 0,85 & 0,30 & 0,33 & 0,48 & 0,84 & $0,64^{*}$ \\
\hline $\mathrm{C}_{14}$ & $-2,03$ & 1,98 & $-0,22$ & $-6,83$ & 5,69 & 1,93 & $-4,24$ & $-0,63$ & $-0,33$ & $-0,02$ & 1,29 & $0,69^{\text {ns }}$ \\
\hline $\mathrm{C}_{15}$ & $-1,61$ & 1,00 & $-1,07$ & $-3,42$ & 0,20 & 6,71 & 0,58 & $-1,00$ & $-0,21$ & $-0,55$ & 0,72 & $0,74^{\mathrm{ns}}$ \\
\hline
\end{tabular}

'a: comprimento do pseudocaule; b: número de folhas por pseudocaule; c: diâmetro do pseudocaule; d: diâmetro do bulbo; e: altura do bulbo; f: peso do bulbo; g: relação diâmetro/altura do bulbo; h: produção total de bulbos; i: forma do bulbo; j: porcentagem de florescimento; 1 : porcentagem de bulbos podres (a: length of the pseudostem, b: number of leaves per pseudostem c: pseudostem diameter, d: diameter of the bulb, e: height of the bulb; f: weight of the bulb; g: diameter/height ratio of the bulb; h: total production of bulbs; i: shape of the bulb; $\mathrm{j}$ : percentage of flowering; 1: percentage of rotten bulbs).

${ }^{2} C_{1}$ : Super Super Precoce (Lages) x Super Super Precoce (Ituporanga); $C_{2}$ : Bela Vista (Lages) x Bela Vista (Ituporanga); $C_{3}$ :Epagri 363 Super Precoce (Lages) x Epagri 363 Super Precoce (Ituporanga); $\mathrm{C}_{4}$ :Baia Indaial (Lages) x Baia Indaial (Ituporanga); $\mathrm{C}_{5}$ : Empasc 352 Bola Precoce (Lages) x Empasc 352 Bola Precoce (Ituporanga); $\mathrm{C}_{6}$ : Empasc 352 Bola Precoce (Lages) x Empasc 355 Juporanga (Ituporanga); $\mathrm{C}_{7}$ : Epagri 362 Crioula Alto Vale (Lages) x Epagri 362 Crioula Alto Vale (Ituporanga); $\mathrm{C}_{8}$ : Bella Catarina (Lages) x Bella Catarina (Ituporanga); $\mathrm{C}_{9}$ : Bella Vista (Lages) x Bella Vista (Ituporanga); $\mathrm{C}_{10}$ : Bella Dura (Lages) x Bella Dura (Ituporanga); $\mathrm{C}_{11}$ : Boreal (Lages) x Boreal (Ituporanga); $\mathrm{C}_{12}$ : Catarina (Lages) x Catarina (Ituporanga); $\mathrm{C}_{13}$ : Crioula Roxa (Lages) x Crioula Roxa (Ituporanga); $\mathrm{C}_{14}$ : Crioula Branca (Lages) x Crioula Branca (Ituporanga); $\mathrm{C}_{15}$ : Gauchinha (Lages) x Gauchinha (Ituporanga). 
Tabela 3. Estimativa da contribuição das variáveis analisadas para a dissimilaridade dos genótipos de cebola (R-square), utilizando o procedimento Stepdisc do SAS nos locais Ituporanga e Lages e probabilidade de erro a 5\% de significância pelo teste $\mathrm{F}$ (estimation of variables contribution to dissimilarity in studied onion genotypes (R-square) using the Stepdisc SAS procedure at Ituporanga and Lages locations. The error probability used was 5\% by $\mathrm{F}$ test). Lages, UDESC/Epagri, 2011.

\begin{tabular}{llcc}
\hline Local & \multicolumn{1}{c}{ Variável } & R-square & Pr>F \\
\hline & Produção total de bulbos & 0,8655 & 0,0001 \\
& Comprimento do pseudocaule & 0,8484 & 0,0001 \\
& Altura do bulbo & 0,7862 & 0,0001 \\
& Porcentagem de florescimento & 0,7416 & 0,0001 \\
& Folhas por pseudocaule & 0,7367 & 0,0001 \\
Ituporanga & Porcentagem de bulbos podres & 0,6028 & 0,0144 \\
& Diâmetro do bulbo & 0,5876 & 0,0263 \\
& Peso do bulbo & 0,6363 & 0,0120 \\
& Diâmetro do pseudocaule & 0,5889 & 0,0428 \\
& Forma do bulbo & 0,6019 & 0,0436 \\
& Relação diâmetro/altura do bulbo & 0,5588 & 0,1099 \\
& Porcentagem de florescimento & 0,8482 & 0,0001 \\
& Peso do bulbo & 0,8111 & 0,0001 \\
& Comprimento do pseudocaule & 0,6939 & 0,0008 \\
& Porcentagem de bulbos podres & 0,6936 & 0,0012 \\
Lages & Relação diâmetro/altura do bulbo & 0,7254 & 0,0006 \\
& Folhas por pseudocaule & 0,7086 & 0,0016 \\
& Diâmetro do pseudocaule & 0,5299 & 0,1114 \\
& Altura do bulbo & 0,5513 & 0,0996 \\
\hline
\end{tabular}

$\mathrm{C}_{15}$ (contraste do genótipo Gauchinha nos dois ambientes), que apresentaram comportamento semelhante nos dois locais (Tabela 1). Tal resultado pode indicar um efeito significativo da interação genótipos $\mathrm{x}$ ambientes para a maioria dos genótipos avaliados e que os genótipos "Crioula Branca" e "Gauchinha" podem ser úteis no desenvolvimento de novas variedades que apresentem ampla adaptação às diferentes condições edafoclimáticas frequentemente encontradas no estado de Santa Catarina.

Ainda assim, nem todos os resultados devem ser considerados completamente elucidados, e podem ser questionadas quais as variáveis que contribuíram de modo relevante para a significância da variação entre os vetores de médias dos tratamentos avaliados. A partir dos valores dos coeficientes canônicos padronizados (CCP) foi possível identificar quais variáveis contribuíram para significância dos contrastes realizados (Tabela 2). Pode ser observado que, nos contrastes realizados, as variáveis nú- mero de folhas por pseudocaule, altura do bulbo, peso do bulbo e porcentagem de bulbos podres apresentaram valores positivos. Por sua vez, as variáveis comprimento do pseudocaule, diâmetro do pseudocaule, diâmetro do bulbo, relação diâmetro: altura do bulbo, produção total de bulbos, forma do bulbo e porcentagem de florescimento apresentaram valores negativos ou valores positivos muito baixos. Valores positivos para os CCP indicam efeito de diferenciação entre os tratamentos, enquanto os valores negativos expressam a similaridade.

Portanto, valores positivos indicam que a característica analisada contribui para que os genótipos apresentem comportamento diferenciado em cada ambiente. Por outro lado, valores negativos indicam que a característica contribuiu para que os contrastes não fossem significativos, ou seja, a característica apresentou uma expressão fenotípica semelhante nos dois ambientes. Pode-se observar também que os resultados apresentados na tabela 2 explicam a significância dos contrastes realizados. Por exemplo, o contraste da cultivar Crioula Branca $\left(\mathrm{C}_{14}\right)$ e o contraste da cultivar Gauchinha $\left(\mathrm{C}_{15}\right)$ apresentaram valores negativos para a maioria das características avaliadas.

Como o efeito da interação genótipos $\mathrm{x}$ ambientes foi significativo, a expressão fenotípica de pelo menos uma das características avaliadas foi diferente para os dois locais (Tabela 3). Por exemplo, para o local Ituporanga as variáveis produção total de bulbos e comprimento do pseudocaule tiveram a maior contribuição na dissimilaridade entre os genótipos. No ambiente Lages os caracteres porcentagem de florescimento e peso do bulbo foram os que apresentaram maior contribuição para a dissimilaridade. Portanto, em estudos de divergência genética, a escolha das características a serem avaliadas, muitas vezes pode não apresentar a mesma relevância em diferentes ambientes.

Dentre os diversos fatores de ambiente que podem ter contribuído para o resultado diferenciado nos dois locais pode-se destacar a influência da temperatura, que se manifesta em diversos aspectos da cultura: seja na capacidade de absorção de nutrientes, no ciclo e na indução do florescimento. De acordo com a classificação climática do estado de Santa Catarina (Köppen), a classificação climática de Ituporanga é de Clima Subtropical Úmido (Cfa) onde a temperatura média do mês mais quente é maior que $22^{\circ} \mathrm{C}$ e a temperatura média do mês mais frio situa-se entre 10 e $15^{\circ} \mathrm{C}$. Já para Lages a classificação climática é de Clima Temperado Úmido (Cfb), onde a temperatura média do mês mais quente é menor do que $22^{\circ} \mathrm{C}$ e a temperatura média do mês mais frio situa-se entre -3 e $18^{\circ} \mathrm{C}$. Segundo Costa et al. (2002), sob condições prolongadas de temperaturas baixas, em torno de $12^{\circ} \mathrm{C}$, pode ser induzido o florescimento prematuro. Tal fato pode ter ocasionado no ambiente Lages, em virtude das baixas temperaturas, o caráter florescimento ter maior contribuição para a variabilidade total do experimento.

A característica peso do bulbo, no ambiente Lages, teve significativa contribuição para a divergência dos genótipos. Este fato pode ser explicado 
Tabela 4. Divergência genética de genótipos de cebola avaliada pela distância de Mahalanobis considerando dois ambientes (Ituporanga e Lages) distintos (genetic divergence of onion genotypes evaluated by the Mahalanobis distance, considering two distinct environments: Lages and Ituporanga). Lages, UDESC/Epagri, 2011.

\begin{tabular}{|c|c|c|c|c|c|c|c|c|}
\hline \multirow{2}{*}{ Genótipo $^{1}$} & \multicolumn{2}{|l|}{ Local } & \multirow{2}{*}{ Genótipo $^{1}$} & \multicolumn{2}{|l|}{ Local } & \multirow{2}{*}{ Genótipo $^{1}$} & \multicolumn{2}{|l|}{ Local } \\
\hline & Ituporanga & Lages & & Ituporanga & Lages & & Ituporanga & Lages \\
\hline $1-2$ & 1,33 & 2,06 & $3-12$ & $2,32 *$ & $5,87^{*}$ & $7-9$ & $3,77^{*}$ & $9,21 *$ \\
\hline $1-3$ & $6,51 *$ & $5,42 *$ & $3-13$ & $17,07 *$ & $6,77^{*}$ & $7-10$ & $14,41 *$ & $11,70 *$ \\
\hline $1-4$ & $3,62 *$ & 1,01 & $3-14$ & $19,95^{*}$ & $7,52 *$ & $7-11$ & $10,16^{*}$ & $16,41 *$ \\
\hline $1-5$ & $3,67 *$ & 0,95 & $3-15$ & $5,95^{*}$ & $10,21^{*}$ & $7-12$ & $5,99 *$ & $7,22 *$ \\
\hline $1-6$ & $12,48^{*}$ & $10,75^{*}$ & $4-5$ & $5,05^{*}$ & 0,73 & $7-13$ & $21,60^{*}$ & $6,38^{*}$ \\
\hline $1-7$ & $13,87^{*}$ & $13,84^{*}$ & $4-6$ & $7,42 *$ & $6,75^{*}$ & $7-14$ & $24,09^{*}$ & $6,76^{*}$ \\
\hline $1-8$ & $18,59 *$ & 0,75 & $4-7$ & $9,08 *$ & $9,47 *$ & $7-15$ & $10,08^{*}$ & $9,70 *$ \\
\hline $1-9$ & $8,75^{*}$ & 2,48 & $4-8$ & $10,03 *$ & 1,46 & $8-9$ & $10,81 *$ & $3,29 *$ \\
\hline $1-10$ & $2,84^{*}$ & $3,92 *$ & $4-9$ & $5,91^{*}$ & 1,24 & $8-10$ & $19,82^{*}$ & $4,52 *$ \\
\hline $1-11$ & $2,46^{*}$ & $2,45^{*}$ & $4-10$ & $3,08 *$ & $2,38 *$ & $8-11$ & $14,31 *$ & $5,15^{*}$ \\
\hline $1-12$ & $3,75^{*}$ & $3,33 *$ & $4-11$ & 2,23 & $2,44^{*}$ & $8-12$ & $15,24 *$ & $4,17^{*}$ \\
\hline $1-13$ & $9,63^{*}$ & $8,14^{*}$ & $4-12$ & $4,30 *$ & 2,19 & $8-13$ & $38,16^{*}$ & $7,53^{*}$ \\
\hline $1-14$ & $8,20 *$ & $7,74^{*}$ & $4-13$ & $19,02 *$ & $5,98 *$ & $8-14$ & $37,52 *$ & $6,69 *$ \\
\hline $1-15$ & $5,49 *$ & $7,04 *$ & $4-14$ & $18,10^{*}$ & $5,66^{*}$ & $8-15$ & $21,03 *$ & 7,11 * \\
\hline $2-3$ & $4,11 *$ & $6,16^{*}$ & $4-15$ & $8,82^{*}$ & $5,85^{*}$ & $9-10$ & $7,73^{*}$ & 2,03 \\
\hline $2-4$ & $4,45^{*}$ & 1,73 & $5-6$ & $9,20 *$ & $7,84^{*}$ & $9-11$ & $4,37 *$ & $2,38 *$ \\
\hline $2-5$ & 1,62 & 1,74 & $5-7$ & $12,62 *$ & $11,15^{*}$ & $9-12$ & 1,62 & 1,25 \\
\hline $2-6$ & 9,92 & $10,32 *$ & $5-8$ & $23,68 *$ & 2,26 & $9-13$ & $15,63 *$ & $5,90 *$ \\
\hline $2-7$ & $11,14^{*}$ & $14,70^{*}$ & $5-9$ & $6,07 *$ & 0,83 & $9-14$ & $17,57^{*}$ & $4,90 *$ \\
\hline $2-8$ & $20,83^{*}$ & $3,21 *$ & $5-10$ & 2,31 & 1,86 & $9-15$ & $4,75^{*}$ & $3,35^{*}$ \\
\hline $2-9$ & $5,95^{*}$ & $2,93 *$ & $5-11$ & $2,96^{*}$ & 1,09 & $10-11$ & $3,07 *$ & $3,45^{*}$ \\
\hline $2-10$ & $2,90 *$ & $2,88^{*}$ & $5-12$ & $2,47 *$ & 1,05 & $10-12$ & $4,33^{*}$ & 2,02 \\
\hline $2-11$ & 1,60 & $3,30 *$ & $5-13$ & $8,24 *$ & $6,29 *$ & $10-13$ & $11,04 *$ & $8,48 *$ \\
\hline $2-12$ & 1,61 & $3,97^{*}$ & $5-14$ & $9,49 *$ & $5,93^{*}$ & $10-14$ & $10,11 *$ & $4,92 *$ \\
\hline $2-13$ & $7,98^{*}$ & $11,23 *$ & $5-15$ & $3,77^{*}$ & $4,37^{*}$ & $10-15$ & $6,34^{*}$ & $4,64^{*}$ \\
\hline $2-14$ & $7,64 *$ & $9,43^{*}$ & $6-7$ & 1,69 & $0,79^{*}$ & $11-12$ & 1,78 & $2,73^{*}$ \\
\hline $2-15$ & $2,77^{*}$ & $8,88^{*}$ & $6-8$ & $10,31 *$ & $9,41 *$ & $11-13$ & $12,78 *$ & $8,95^{*}$ \\
\hline $3-4$ & $4,19 *$ & $3,47^{*}$ & $6-9$ & $3,49 *$ & $5,99 *$ & $11-14$ & $11,94 *$ & $7,88^{*}$ \\
\hline $3-5$ & $4,08 *$ & $5,68 *$ & $6-10$ & $10,48 *$ & $8,40^{*}$ & $11-15$ & $3,36^{*}$ & $5,65^{*}$ \\
\hline $3-6$ & $4,87^{*}$ & $3,36^{*}$ & $6-11$ & $9,37^{*}$ & $12,55^{*}$ & $12-13$ & $9,44 *$ & $4,30^{*}$ \\
\hline $3-7$ & $5,50 *$ & $3,97 *$ & $6-12$ & $5,75^{*}$ & $4,79 *$ & $12-14$ & $10,47^{*}$ & $4,02 *$ \\
\hline $3-8$ & $14,06^{*}$ & $3,84^{*}$ & $6-13$ & $19,38 *$ & $6,03^{*}$ & $12-15$ & 1,89 & 2,28 \\
\hline $3-9$ & $2,91^{*}$ & $5,22 *$ & $6-14$ & $22,01 *$ & $6,30 *$ & $13-14$ & 1,69 & 2,25 \\
\hline $3-10$ & $7,20 *$ & $6,84^{*}$ & $6-15$ & $9,69^{*}$ & $7,98^{*}$ & $13-15$ & $5,89^{*}$ & $3,75^{*}$ \\
\hline $3-11$ & $3,61^{*}$ & $10,06^{*}$ & $7-8$ & $9,26^{*}$ & $11,62 *$ & $14-15$ & 6,71 & $1,86^{*}$ \\
\hline
\end{tabular}

"significativo a $5 \%$ de probabilidade de erro pelo teste $\mathrm{F}$ (significant at $5 \%$ of probability of error by the $\mathrm{F}$ test). ${ }^{1} 1=$ SuperSuper Precoce; 2=Bela Vista; 3= Superprecoce; 4= Baia Indaial; 5= Bola Precoce; 6= Juporanga; $7=$ Crioula; 8= Bella Catarina; 9= Bella Vista; $10=$ Bella Dura; $11=$ Boreal; $12=$ Catarina; $13=$ Crioula Roxa; $14=$ Crioula Branca; $15=$ Gauchinha.

em função da análise de solo apresentar valores elevados para a maioria dos nutrientes e assim favorecer o desenvolvimento dos bulbos dos genótipos com maior potencial produtivo. Já para o ambiente Ituporanga a produção to- tal de bulbos, que apresentou o maior valor, pode ter sido ocasionada por um número de plantas por parcela diferente, em função de perdas de plantas ou mesmo plantio não uniforme. Portanto esta característica precisa ser melhor avaliada em outros trabalhos. O caráter comprimento do pseudocaule teve uma contribuição importante na dissimilaridade dos genótipos nos dois locais, em discordância com resultados obtidos por Buzar et al. (2007). Tal fato pode 
ser explicado pelo fato deste autor ter avaliado características diferentes das apresentadas neste trabalho e também pela influência do ambiente.

No sentido de verificar a divergência entre os genótipos, foi utilizada a distância de Mahalanobis como medida de dissimilaridade (Tabela 4). Trata-se da mais robusta das medidas de distância disponíveis para a análise de dados quantitativos, sendo referida em vários trabalhos como distância genética, quando aplicada em dados experimentais (Dias, 2006). Houve diferenças nas medidas de dissimilaridade entre os dois ambientes. Em Ituporanga os valores foram maiores, chegando a 38,16 entre os genótipos "Crioula Roxa" e "Bella Catarina". Doze medidas de distâncias entre os genótipos tiveram valores não significativos. Já em Lages o maior valor foi de 16,41 entre os genótipos "Boreal" e "Epagri 362 Crioula Alto Vale" e 21 medidas de distância não foram significativas. Diante destes resultados pode-se dizer que o ambiente Ituporanga discriminou melhor os genótipos.

Desta maneira pode ser concluído que, para o ambiente Ituporanga, os cruzamentos entre o genótipo "Bella Catarina" e "Crioula Roxa" ou "Crioula Branca" podem proporcionar uma população segregante com constituições genéticas de interesse. Visto que estes dois últimos genótipos não apresentam boas características agronômicas, pode-se recomendar o cruzamento da "Bella Catarina" com os genótipos "Gauchinha", "Catarina" e "Boreal", que são cultivares com boa aceitação comercial. Já para o ambiente Lages os cruzamentos mais divergentes seriam entre os genotipos "Boreal" e "Epagri 362 Crioula Alto Vale" ou "Boreal" e "Empasc 355 Juporanga". Outra possibilidade seria o cruzamento entre "Epagri 362 Crioula Alto Vale" e "Bela Vista".

As diferenças significativas encontradas entre os genótipos de cebola cultivados no estado de Santa Catarina indicam que existe variabilidade dispo- nível para ser utilizada em programas de melhoramento da cultura. Ampla variabilidade genética da coleção de germoplasma de cebola também foi observada por Santos et al. (2011). Os caracteres analisados sofreram forte influência do ambiente, com reflexo direto sobre o desempenho dos genótipos estudados, independente do local avaliado. Os caracteres que mais contribuíram para a dissimilaridade entre os genótipos foram produção total de bulbos e comprimento do pseudocaule para o ambiente Ituporanga e porcentagem de florescimento e peso do bulbo para o ambiente Lages. Os genótipos "Crioula Branca" e "Gauchinha" podem ser úteis no desenvolvimento de variedades que apresentem ampla adaptação às diferentes condições edafoclimáticas de Santa Catarina.

\section{REFERÊNCIAS}

BARBIERI RL; LEITE DL; CHOER E; SINIGAGLIA C. 2005. Divergência genética entre populações de cebola com base em marcadores morfológicos. Ciência Rural 35: 303-308.

BOITEUX LS; MELO PCT. 2004. Taxonomia e origem. In: EMBRAPA HORTALIÇAS. Sistema de produção de cebola (Allium cepa L.). Brasília: Embrapa-CNPH. Sistemas de Produção. Versão Eletrônica.

BORÉM A; MIRANDA GV. 2009. Melhoramento de plantas. Viçosa-MG: Ed. UFV, 529p.

BUZAR AGR; OLIVEIRA VR; BOITEUX LS. 2007. Estimativa da diversidade genética de germoplasma de cebola via descritores morfológicos, agronômicos e bioquímicos. Horticultura Brasileira 25: 527-532.

COIMBRA JLM; BERTOLDO JG; ELIAS HT; HEMP S; VALE NM; TOALDO D; ROCHA F; BARILI LD; GARCIA SH; GUIDOLIN AF; KOPP MM. 2009. Mineração da interação genótipo $\mathrm{x}$ ambiente em Phaseolus vulgaris L. para o Estado de Santa Catarina. Ciência Rural 39: 355-363.

COIMBRA JLM; SANTOS JCP; ALVES MV; BARZOTTO I. 2007. Técnicas multivariadas aplicadas ao estudo da fauna do solo: contrastes multivariados e análise canônica discriminante. Revista Ceres 54: 270-276.

COSTA ND; LEITE DL; SANTOS CAF; CANDEIA JA; VIDIGAL SM. 2002. Cultivares de cebola. Informe Agropecuário 23: 20-27.

CRUZ CD; REGAZZI AJ. 2001. Programa Genes: Aplicativo computacional em genética e estatística. Viçosa: Editora UFV. 648p.

CRUZ CD; CARNEIRO PCS. 2003. Modelos biométricos aplicados ao melhoramento genético. Viçosa: Editora UFV. 579p.

CUI Z; CARTER JR TE; BURTON JW; WELLS R. 2001 Phenotypic diversity of modern Chinese and North American soybean cultivars. Crop Science 41: 1954-1967.

DIAS LAS; 2006. Análises Multidimensionais. In: ALFENAS AC. Eletroforese e marcadores bioquímicos em plantas e microorganismos. 2.ed. Viçosa: Editora UFV. Cap. 9, p.405-475.

EPAGRI. Sistema de produção para cebola: Santa Catarina. 2000. Florianópolis. 91p.

FERREIRA DF. 1996. Análise Multivariada. Lavras. 388p.

HEIDEMANN JC; ANDRADE LRB; STÄHELIN D; PREZZI HA; VALENTINI G; BERTOLDO JG; GUIDOLIN AF; COIMBRA JLM. 2010. Variabilidade em acessos do banco ativo de Germoplasma de feijão do CAV/UDESC. In: Congresso de Iniciação Científica de PósGraduação. Florianópolis. Anais eletrônicos. Florianópolis: UDESC, 2010. Disponível em: $<$ http://www.udesc.br/arquivos/secao/proppg/ Seminario20/posteres_agrarias.pdf $>$. Acesso em: 12 jul. 2011.

IPGRI, ECP/GR, AVRDC. 2001. Descriptors for Allium (Allium spp.). Rome: International Plant Genetic Resources Institute, European Cooperative for Crop Genetic Resources Networks (ECP/GR); Taiwan: Asian Vegetable Research and Development Center. 43p.

LEITE DL. 2007. Melhoramento genético de cebola. In: BARBIERI RL (ed). Cebola: ciência, arte e história. 2. ed. Brasília: Embrapa Informação Tecnológica. p.79-113.

MARIOT MP; BARBIERI RL; SINIGAGLIA C; RIBEIRO MV. 2008. Variabilidade em matrizes de acessos de espinheira-santa. Ciência Rural 38: 351-357.

MOURA WM; CASALI VWD; CRUZ CD; LIMA PC. 1999. Divergência genética em linhagens de pimentão em relação à eficiência nutricional de fósforo. Pesquisa Agropecuária Brasileira 34: 217-224.

OLIVEIRAVR; MENDONÇAJL; SANTOS CAF. 2004. Clima. In: EMBRAPA HORTALIÇAS. Sistema de produção de cebola (Allium cepa L.). Brasília: Embrapa-CNPH. Sistemas de Produção. Versão Eletrônica.

SAS INSTITUTE INC. (2007). SAS ${ }^{\circledR}$ 9.1.3 (TS1M3) for Windows Microsoft. Cary, NC, SAS Institute Inc. 212p.

SANTOS CAF; OLIVEIRA VR; RODRIGUES MA; RIBEIRO HLC; SILVA GO. 2011. Similaridade genética entre cultivares de cebola de diferentes tipos e origens, baseada em marcadores AFLP. Horticultura Brasileira [online]. 29: 32-37.

SCHMITT DR. 2010. Síntese Anual da Agricultura de Santa Catarina: Empresa de Pesquisa Agropecuária e Extensão Rural/Centro de Socioeconomia e Planejamento Agrícola Epagri/Cepa, Florianópolis-SC. p. 77-81. 